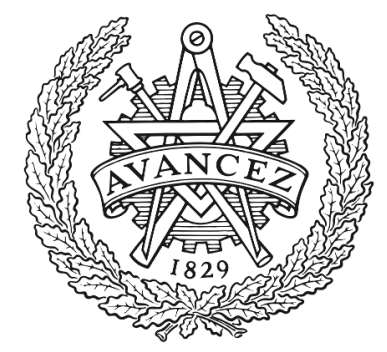

CHALMERS

UNIVERSITY OF TECHNOLOGY

\title{
Plasmonic Metasurface for Spatially Resolved Optical Sensing in Three Dimensions
}

Downloaded from: https://research.chalmers.se, 2023-04-26 14:38 UTC

Citation for the original published paper (version of record):

Nugroho, F., Albinsson, D., Antosiewicz, T. et al (2020). Plasmonic Metasurface for Spatially

Resolved Optical Sensing in Three Dimensions. ACS Nano, 14(2): 2345-2353.

http://dx.doi.org/10.1021/acsnano.9b09508

N.B. When citing this work, cite the original published paper. 


\section{Plasmonic Metasurface for Spatially Resolved Optical Sensing in Three Dimensions}

Ferry Anggoro Ardy Nugroho,* David Albinsson, Tomasz J. Antosiewicz, and Christoph Langhammer*

Cite This: ACS Nano 2020, 14, 2345-2353

Read Online

ACCESS | Lلll Metrics \& More | 国 Article Recommendations ｜ sl Supporting Information

ABSTRACT: The highly localized sensitivity of metallic nanoparticles sustaining localized surface plasmon resonance (LSPR) enables detection of minute events occurring close to the particle surface and forms the basis for nanoplasmonic sensing. To date, nanoplasmonic sensors typically consist of two-dimensional (2D) nanoparticle arrays and can therefore only probe processes that occur within the array plane, leaving unaddressed the potential of sensing in three dimensions (3D). Here, we present a plasmonic metasurface comprising arrays of stacked Ag nanodisks separated by a thick $\mathrm{SiO}_{2}$ dielectric layer, which, through rational design, exhibit two distinct and spectrally separated LSPR sensing peaks and corresponding

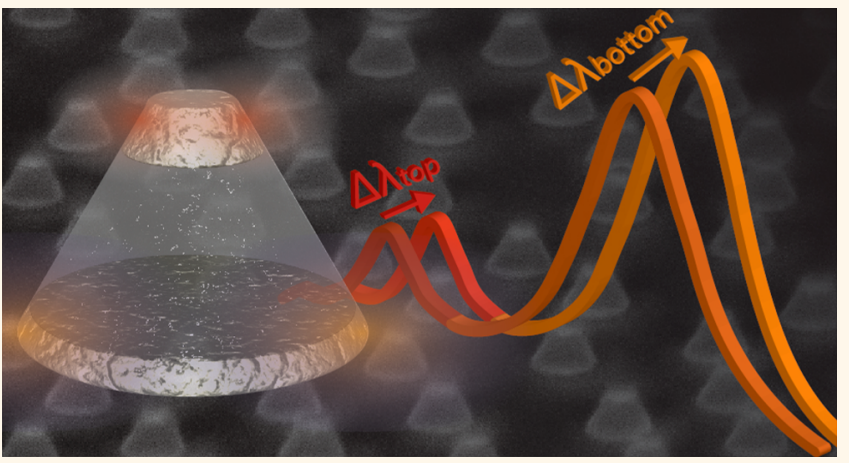
spatially separated sensing locations in the axial direction. This

arrangement thus enables real-time plasmonic sensing in 3D. As a proof-of-principle, we successfully determine in a single experiment the layer-specific glass transition temperatures of a bilayer polymer thin film of poly(methyl methacrylate), PMMA, and poly(methyl methacrylate)/poly(methacrylic acid), P(MMA-MAA). Our work thus demonstrates a strategy for nanoplasmonic sensor design and utilization to simultaneously probe local chemical or physical processes at spatially different locations. In a wider perspective, it stimulates further development of sensors that employ multiple detection elements to generate distinct and spectrally individually addressable LSPR modes.

KEYWORDS: nanoplasmonics, nanoplasmonic sensor, nanofabrication, 3D, polymer, glass transition temperature, materials science

$\mathrm{T}$ he localized surface plasmon resonance (LSPR) ${ }^{1}$ occurring in metal nanoparticles forms the basis for applications that include photocatalysis, ${ }^{2,3}$ surfaceenhanced spectroscopy, ${ }^{4,5}$ and sensing., ${ }^{6,7}$ Particularly for sensing, LSPR functionality is enabled by the sensitivity of the enhanced electromagnetic field extending from the nanoparticle surface to a change in the refractive index (RI) of the surrounding medium. Furthermore, the fact that the enhanced field decays very rapidly ${ }^{8}$ offers a unique situation where the LSPR only probes a minute volume (i.e., the sensing volume) in close vicinity of the particle surface and thus allows detection of a wide range of processes that include molecular interactions and material transformation within that volume. Ever since the first demonstration of the nanoplasmonic sensing concept by Englebienne ${ }^{9}$ two decades ago, it has developed into a major subfield of plasmonics and has found wide applications such as in bio- and chemosensing ${ }^{6,7,10,11}$ and in materials science. ${ }^{12,13}$ This, in turn, has spurred the development of a large library of plasmonic nanostructures tailored for various sensing purposes. ${ }^{6,14-16}$ However, as a review of the corresponding literature shows, nanoplasmonic sensors to the largest extent only comprise two-dimensional
(2D) arrays of a single type of plasmonic particle on a support. Consequently, they can only detect processes that occur within the plane of the array. This leaves unaddressed the potentially widely relevant concept of plasmonic sensors that could simultaneously probe multiple locations in three-dimensional (3D) space. The implementation of such a concept would, however, unlock access to intriguing opportunities, such as to accurately and simultaneously scrutinize spatially varying processes occurring at the nanoscale in $3 \mathrm{D}$. This is of high interest, for example, in nanomaterials science because material properties often are locally unique and may depend on the spatial location inside the material. ${ }^{17}$ Considering the traits of nanoplasmonics, in principle, such a sensing concept could be enabled by distributing in 3D geometrically dissimilar plasmonic particles that exhibit LSPR at different wavelengths, to render them spectrally addressable simultaneously. How-

Received: December 3, 2019

Accepted: January 27, 2020

Published: January 27, 2020 
ever, to the best of our knowledge, such a 3D plasmonic sensing concept has never been realized, despite a number of works that demonstrate plasmonic sensors comprising multiantennas ${ }^{18-21}$ or active plasmonic particles protruding far away from the support. ${ }^{2,23}$

Here, to introduce such a 3D sensing platform conceptually envisioned above, we present a plasmonic metasurface which consists of a quasi-random array of nanoarchitectures comprising two plasmonic nanoparticles with spectrally separated resonance wavelengths. The plasmonic nanoparticles are separated vertically by a thick dielectric layer in a stack that protrudes from the support. As we show using both experiments and finite-difference time-domain (FDTD) simulations, this enables simultaneous and independent sensing at the corresponding plasmonic particle locations in the 3D stack. To demonstrate the functionality of the sensor in a materials science application recently introduced, ${ }^{24-29}$ we simultaneously characterize the layer-specific glass transition temperatures of stacked poly(methyl methacrylate), PMMA, and poly(methyl methacrylate)/poly(methacrylic acid), P(MMA-MAA), copolymer thin films in a single experiment.

\section{RESULTS AND DISCUSSION}

We designed the metasurface to comprise a quasi-random array of truncated $\mathrm{SiO}_{2}$ nanocone structures, which contain vertically separated and differently sized plasmonically active Ag nanodisks at their base and at their tip (Figure 1a). These disks then function as independent antennas that exclusively probe their particular surroundings. This independent function requires sufficient spatial separation between the disks to minimize near-field coupling, and that they exhibit LSPR spectrally separated enough that two independent "peaks" can be resolved in their optical spectrum. With respect to the latter requirement, we aimed to have both LSPR peaks within the visible to near-infrared (NIR) spectral range (i.e., 400-1100 $\mathrm{nm}$ ) to comply with the most commonly used optical components in the field. ${ }^{6}$ With this constraint at hand, the choice of the metal for the sensing antennas is practically restricted to Ag, which features a narrow LSPR peak and a high interband absorption onset $(3.8 \mathrm{eV}$ or $\sim 325 \mathrm{~nm}$, i.e., beyond the designed wavelength range).$^{30-32}$ This is superior to $\mathrm{Au}$, which exhibits broader LSPR modes and has a lower interband transition threshold energy $(2.3 \mathrm{eV}$ or $\sim 540 \mathrm{~nm}) .^{30,33}$ Utilizing $\mathrm{Ag}$ thus provides more room for the design of the antennas' dimensions while maximizing their spatial separation in order to produce two spectrally well-separated LSPR peaks.

To fabricate this sensor architecture, we employed the holemask colloidal lithography (HCL) nanofabrication method. ${ }^{34}$ Due to the fact that the rim of the mask shrinks during material deposition, ${ }^{35}$ HCL fabrication generates tapered, instead of straight-walled, nanostructures. This provides a situation where subsequent deposition of metal-dielectric-metal multilayers through the mask naturally produces vertically separated metal nanodisks with different diameter (hence different LSPR wavelength), which is directly controlled by the chosen vertical separation in the stack. Additionally, fine-tuning of the exact resonance wavelength of each antenna is controlled by its thicknesses. For the present case, we have optimized these structures such that the corresponding metasurface comprises a quasi-random array of truncated nanocones. They have an average base diameter of $210 \mathrm{~nm}$ that corresponds to the first Ag nanodisk ("bottom") with $20 \mathrm{~nm}$ thickness, separated by 90 $\mathrm{nm} \mathrm{SiO}_{2}$ from a second $\mathrm{Ag}$ disk at the tip of the structure
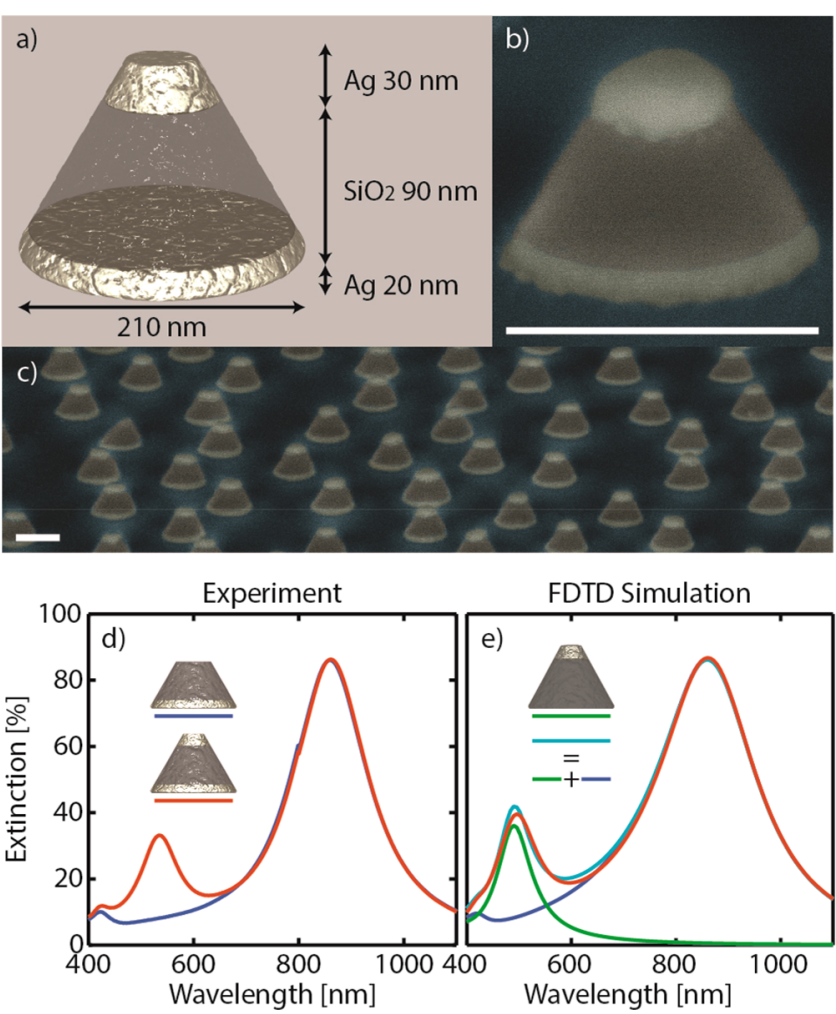

Figure 1. Three-dimensional metasurface architecture and optical spectra. (a) Artist's rendition of the 3D nanoplasmonic sensor architecture. Two Ag nanodisks of different diameter and thickness optimized to maximize spectral separation of their LSPR are vertically separated by a thick $\mathrm{SiO}_{2}$ spacer layer. Tilted SEM image of a (b) single and (c) quasi-random array of a 3D sensor metasurface. Scale bars are $200 \mathrm{~nm}$. (d) Experimental optical extinction spectra of metasurfaces comprising 3D nanostructures with (red) and without (blue) the top disk. Two spectrally well-separated peaks are apparent for the $3 \mathrm{D}$ sensor, corresponding to the top (short wavelengths) and bottom (long wavelengths) disks. Absence of the top disk in the $3 \mathrm{D}$ structures (blue) only results in the absence of the short wavelengths peak, whereas the peak corresponding to the bottom disk is unaffected, corroborating little to no crosstalk between the top and bottom disks. (e) FDTD-simulated optical extinction spectra of 3D sensors, corroborating the experimental findings presented in (d). Also shown are the extinction spectra of a sensor with only top disk (green) and sum of the extinction of the sensor with only bottom disk and sensor with only top disk (cyan).

("top") with a diameter of $\sim 80 \mathrm{~nm}$ and a thickness of $30 \mathrm{~nm}$ (Figure 1a-c).

Measuring the optical extinction spectrum of a metasurface containing these nanostructures reveals the intended distinct and well-separated two LSPR peaks within the targeted wavelength range: one at $860 \mathrm{~nm}$ (bottom disk) and another at $535 \mathrm{~nm}$ (top disk; Figure 1d). To confirm that the disks are not coupled via their near-fields, we also fabricated an identical metasurface with the bottom disk and cone structure but without the top disk. As shown in Figure 1d, the extinction spectrum of such a system is identical except for the short wavelength range where the top disk LSPR peak is lacking. In other words, removing (or adding) the top disk from (to) the $3 \mathrm{D}$ structure does not alter the optical response of the bottom disk. Also, increasing the spatial separation between bottom and top disk to $100 \mathrm{~nm}$ only results in the change of the top disk LSPR peak (due to smaller diameter), whereas the 
response of the bottom disk remains, for practical purpose, unchanged (Figure S1). This contrasts reported cases of coupled systems, where spectral features that depend on a nonnegligible coupling strength and not only on the constituent antennas can be observed ${ }^{36,37}$ (see also Figure S1 where we shorten the distance between the disks to $60 \mathrm{~nm}$ ). Furthermore, FDTD simulations of a single 3D nanostructure reproduce accurately the experimental observations and thus further corroborate the lack of near-field coupling between the disks (Figure 1e). We note that the LSPR peak in the simulation is broader than the experimental one, contrary to what one expects due to the size variation in individual nanostructures in ensemble measurements. We recall that the optical response of our amorphous arrays (quasi-random with short-range order) is determined by the single-particle response which, however, is modified by the stochastically scattered fields by other nanoparticles. ${ }^{38,39}$ The magnitude and sign of the stochastic interference of the scattered fields with the incident one are determined by the particle minimum center-to-center distance (see Figure S3 for our metasurface). These result in either a red or blue shift of the single-particle resonance position, narrowing or broadening of the peak width, and corresponding change of the amplitude. ${ }^{40,41}$ This interaction is the origin of a majority of the differences between the experimental and simulated response, whereas additional factors originate from using a tabulated permittivity for $\mathrm{Ag}$ which is likely close to, but not exactly equal to, experiments as well a minor geometrical difference between the modeling and experiment.

Having established the metasurface nanoarchitecture as such, we now turn to assessing the Ag antennas for practical sensing applications. As a first step to enable this, we address the fact that $\mathrm{Ag}$ is prone to oxidation by adopting one of the concepts of indirect nanoplasmonic sensing, ${ }^{42}$ in which a thin coating film is applied to the sensing structure in order to protect it from the environment and permit application also in demanding chemical environments as well as at high temperatures. Furthermore, application of such a thin coating also provides a chemically uniform surface, which is of importance in sensing applications where the interaction between the sensor surface and analyte is critical for the behavior of the latter. Here, we choose $\mathrm{Si}_{3} \mathrm{~N}_{4}$ as the coating material due to its low permeability toward oxygen and its high temperature stability. ${ }^{43}$ Specifically, employing plasma-enhanced chemical vapor deposition (PE-CVD), we apply a conformal $10 \mathrm{~nm}$ thin $\mathrm{Si}_{3} \mathrm{~N}_{4}$ coating on the entire metasurface (Figure 2a), which still preserves its distinguishable LSPR peaks (Figure S2).

To evaluate the effectiveness of the $\mathrm{Si}_{3} \mathrm{~N}_{4}$ coating to protect the $\mathrm{Ag}$ nanoparticle elements of the metasurface from oxidizing, we devised an experiment to assess the coated sensor's stability under oxidizing conditions at high temperature. Specifically, we exposed a coated metasurface, along with an uncoated control, to a flow of $2 \% \mathrm{O}_{2}$ in $\mathrm{Ar}$ carrier gas while slowly increasing the temperature to $600{ }^{\circ} \mathrm{C}$ and simultaneously measured the evolution of their extinction spectra (Figure 2b,c). Starting from a comparable extinction spectrum profile, both LSPR peaks of the uncoated sensor blue shift more pronouncedly than the ones of the coated sensor as the temperature increases to $\sim 450{ }^{\circ} \mathrm{C}$. Apart from this minor shift, the intensity of the peaks appears to be unaffected up to this temperature, hinting that the Ag disks are not oxidized. ${ }^{44}$ The observed spectral changes are thus explained by the reshaping
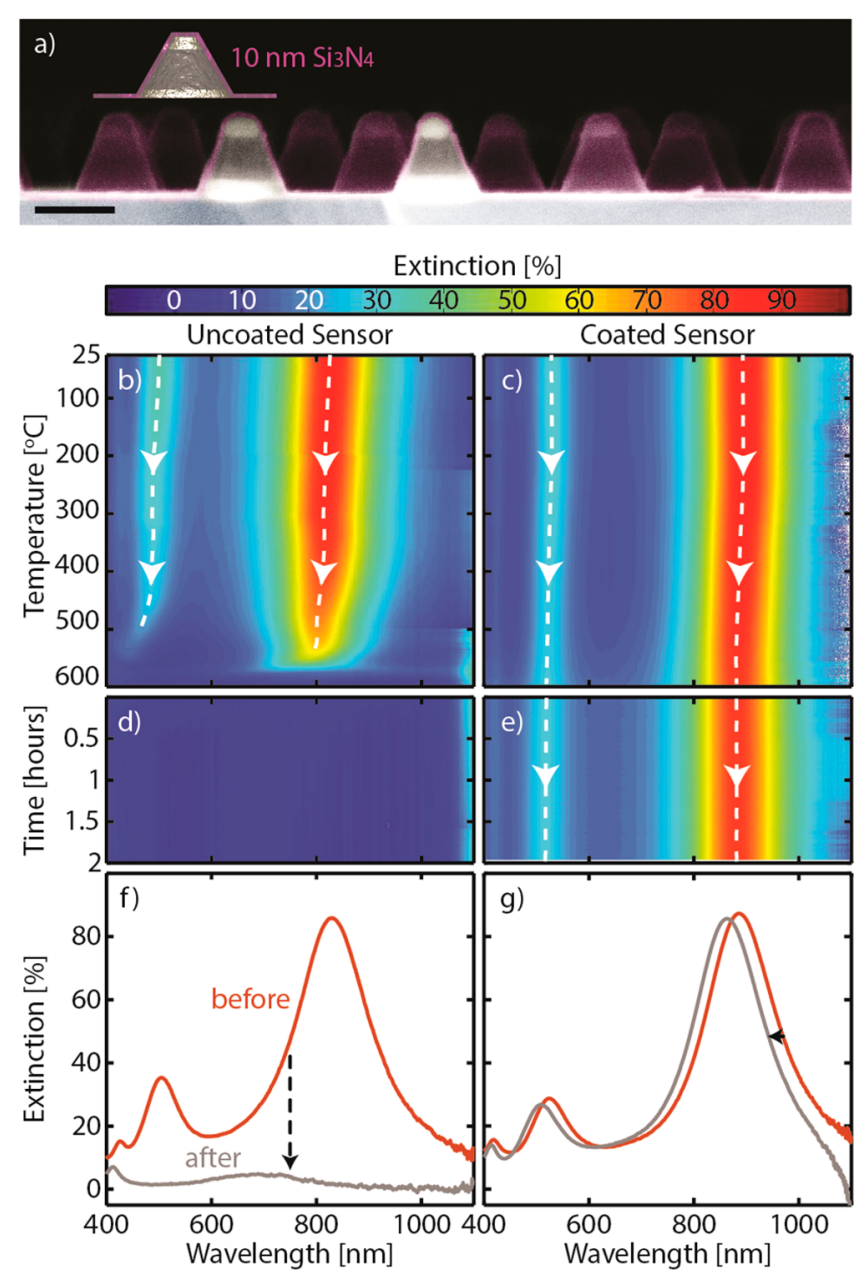

Figure 2. Coating of the 3D metasurface and its stability. (a) Falsecolored SEM image of a 3D sensor plasmonic metasurface coated with a $10 \mathrm{~nm} \mathrm{Si}_{3} \mathrm{~N}_{4}$ layer (violet). Also shown is the to-scale schematic of the coated sensor. Scale bar is $200 \mathrm{~nm}$. Wavelengthresolved temporal evolution of optical extinction of (b) uncoated and (c) $\mathrm{Si}_{3} \mathrm{~N}_{4}$-coated $3 \mathrm{D}$ sensors as a function of increasing temperature up to $600{ }^{\circ} \mathrm{C}$ under constant flow of $2 \% \mathrm{O}_{2}$ in Ar. The dashed lines denote the spectral peak maximum, $\lambda_{\text {peak }}$ of the bottom and top disks. For the uncoated sensor, the bottom and top disk LSPR peaks vanish around 500 and $550{ }^{\circ} \mathrm{C}$, respectively, due to complete oxidation. Extended $2 \mathrm{~h}$ exposure to $2 \% \mathrm{O}_{2}$ in $\mathrm{Ar}$ at $600{ }^{\circ} \mathrm{C}$ is also shown for the (d) uncoated and (e) coated sensors. Evidently, the $\mathrm{Si}_{3} \mathrm{~N}_{4}$ coating excellently protects the $\mathrm{Ag}$ nanodisks from oxidation even at very harsh conditions. $(\mathrm{f}, \mathrm{g})$ Room temperature extinction spectra of an uncoated and coated metasurface, respectively, before and after the heating/oxidation test.

of the sensors induced by heat ${ }^{45}$ (in contrast to heat-induced particle expansion that causes the LSPR red shift in isotropic or thermally stable particles, ${ }^{46,47}$ which we also observed for subsequent heating ramps, after the initial reshaping; see Figure S5), which expectedly happens to a lower degree for the coated system thanks to the good thermal stability of $\mathrm{Si}_{3} \mathrm{~N}_{4}$ that prevents (major) reshaping of the encapsulated Ag disks. When heating progresses to $\sim 500{ }^{\circ} \mathrm{C}$, the optical response from the uncoated top disk abruptly diminishes and is soon followed by the bottom disk at $\sim 550{ }^{\circ} \mathrm{C}$, both signaling the complete oxidation and thus loss of plasmonic response. In contrast, for the coated system, extended heating up to $600{ }^{\circ} \mathrm{C}$ only prolongs the slight blue shift of the LSPR peaks observed 
above, and even with an additional $2 \mathrm{~h}$ of $\mathrm{O}_{2}$ exposure at 600 ${ }^{\circ} \mathrm{C}$, it does not alter its response (Figure $2 \mathrm{~d}-\mathrm{g}$ ). The overall slight blue shift after cooling back to room temperature (Figure $2 \mathrm{~g}$ ) can be assigned to minor reshaping, as confirmed by the scanning electron microscopy (SEM) images taken before and after the experiment (Figure S4).

After confirming the structural and plasmonic integrity of the $\mathrm{Si}_{3} \mathrm{~N}_{4}$-coated metasurface (hereafter referred to as the metasurface), we now turn to characterize its sensing properties. Aided by FDTD simulations, we start by mapping the extension of electromagnetic field enhancement for top and bottom disk elements in air. The corresponding field enhancement profiles calculated by FDTD at the resonance wavelength of the respective disk for normal-incidence plane wave illumination are shown in Figure 3a,b. It becomes clear
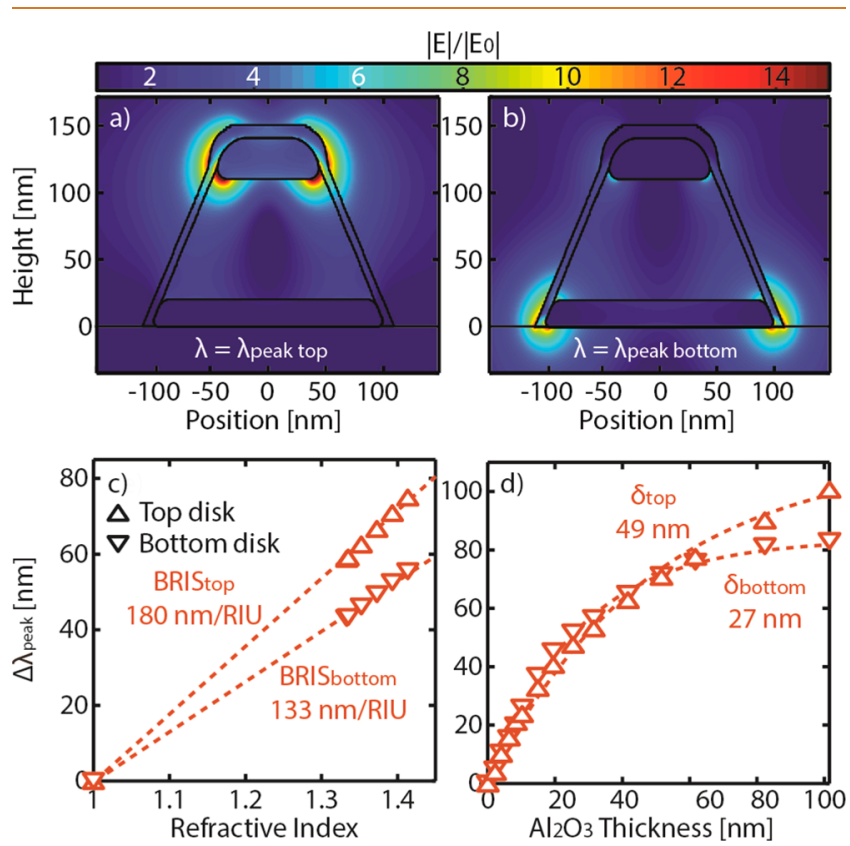

Figure 3. Sensing properties of the 3D metasurface. Electromagnetic field strength distribution for excitation by a normalincidence plane wave at the resonance wavelength of the (a) top and (b) bottom disks in air, normalized to the incoming field strength. Evidently, the near-fields of the top and bottom disks excited at their corresponding LSPR wavelengths do not spatially overlap with the nonexcited particle, corroborating little to no coupling between them and thus that they are able to independently sense a nearby event excited simultaneously at their respective LSPR wavelength. (c) Bulk refractive index sensitivity and (d) enhanced field decay length, $\delta$, in air determination for bottom and top disks. The decay lengths further quantify the non-overlapping fields of the bottom and top disks as their sum is smaller than the distance between the disks (i.e., 76 vs $90 \mathrm{~nm}$ ). Overall, the top disk displays high sensitivity despite its small size, due to a large accessible sensing volume because of its location on a pillar. ${ }^{48}$

that field enhancement is observed exclusively at the position of the resonating $\mathrm{Ag}$ disk element for the two irradiation wavelengths, and that there is no spatial overlap (Figure S6), further corroborating the anticipated independent local sensitivity of each disk. ${ }^{8}$ Shortening the separation between the disks, we found that the fields are coupled for the spacer layer thickness of less than $70 \mathrm{~nm}$ (Figures S7 and S8); hence, it corresponds to the minimum separation distance for the $3 \mathrm{D}$ sensor fabricated using the method and base diameter here to exhibit the independent spatial sensing function. Finally, we also note that the spatial distribution of the field enhancement exhibits an asymmetry, which is a consequence of the higher RI of both the substrate and the $\mathrm{SiO}_{2}$ layer between the disks. ${ }^{48,49}$

We also experimentally quantify two key sensing characteristics of each of the sensor disks, namely, their bulk refractive index sensitivity (BRIS) and their local/thin film sensitivity. The BRIS, expressed as $\mathrm{nm} /$ refractive index unit (RIU), is derived by tracking the peak shifts of the bottom and top disks, $\Delta \lambda_{\text {peak }}$ as the metasurface is exposed to liquid media with varying RI (Figure 3c). Clearly, each disk responds independently and differently (see raw data in Figure S9) and thus exhibits different BRIS (i.e., 133 and $180 \mathrm{~nm} / \mathrm{RIU}$ for bottom and top disks, respectively). To this end, it is interesting to discuss the higher sensitivity of the top disk compared to that of the bottom one despite the smaller size of the former, as it has been shown both experimentally and theoretically that BRIS and plasmonic antenna size are, in general, positively correlated. ${ }^{50,51}$ The exceptionally high sensitivity in the top disk in the present case is a direct consequence of its position on top of a $\mathrm{SiO}_{2}$ pillar, which exposes a larger fraction of its sensing volume compared to a disk located on a flat support ${ }^{48,49}$ (Figure S12). This picture is consistent with the simulated enhanced field mapping discussed above (cf. Figure 3a,b).

To quantify the local or thin film sensitivity of the disks in air, we set out to determine their field decay lengths, $\delta$, experimentally. This decay length marks the distance at which the field intensity decays to $1 / e$ of that of the one on the disk surface. Hence, by definition, it signifies a boundary between the sensing volume within which sensitivity is high and the volume further away from the disk surface within which sensitivity rapidly decreases. ${ }^{52}$ To experimentally determine these decay lengths, we subsequently deposit ultrathin $\mathrm{Al}_{2} \mathrm{O}_{3}$ films by atomic layer deposition and map the $\Delta \lambda_{\text {peak }}$ induced by the deposition of each layer (Figure 3d; see also Supporting Information for the experimental details and derivation). It is clear that the bottom and top disks display similar $\Delta \lambda_{\text {peak }}$ up to $\sim 50 \mathrm{~nm} \mathrm{Al} \mathrm{O}_{3}$. For thicker coatings, the $\Delta \lambda_{\text {peak }}$ of the top disk is higher than that of the bottom one. This is translated to a longer decay length of the top disk $\left(\delta_{\text {top }}=49 \mathrm{~nm}\right)$ compared to that of the bottom one $\left(\delta_{\text {bottom }}=27 \mathrm{~nm}\right)$, which once more is a direct consequence of the top disk position on top of the $\mathrm{SiO}_{2}$ pillar. The derived decay lengths also provide a quantitative validation for the lack of near-field coupling between the disks as their sum is smaller than the distance between the disks (i.e., 76 vs $90 \mathrm{~nm}$ ). This is in very good agreement with the FDTD simulations discussed above (cf. Figure $3 a, b$, and also see Figure S6). In general, the decay lengths become shorter when the metasurface is embedded in a medium with higher RI due to the higher optical density (Figures S10 and S11).

As the final step of our evaluation and as a proof-of-principle of our envisioned 3D sensing functionality, we designed an experiment to extract the thermophysical properties of multilayer polymer thin films by attempting to simultaneously determine the glass transition temperatures, $T_{\mathrm{g}}$, of the individual layers. $T_{\mathrm{g}}$ is the temperature below which a polymer is in a glassy state. ${ }^{53}$ Such thin polymer multilayer systems are of high relevance in organic electronics ${ }^{54,55}$ and organic photovoltaics. ${ }^{56,57}$ In these applications, device performance is strongly affected by the thermal stability of constituent polymers, which in turn is characterized by their $T_{\mathrm{g}}{ }^{58,59}$ For 
our proof-of-principle experiments, we here choose PMMA and its corresponding copolymer P(MMA-MAA) because they possess similar optical properties (transparent in the visibleNIR range and RI of 1.49 , as measured by ellipsometry; see Figure S13) and yet are expected to have different thermophysical properties due to the stronger bonding between the monomers in the copolymer. ${ }^{60,61}$

To prepare the samples, we use a combination of spin coating and plasma etching (see details in Methods and also Figure S14) to subsequently deposit flat (i.e., not conformal to the 3D sensor structure) P(MMA-MAA) and PMMA films, such that each of the polymer layers only encapsulates one of the disks. Specifically, we deposit first $70 \mathrm{~nm}$ of P(MMAMAA), such that its surface levels roughly at the middle of the $\mathrm{SiO}_{2}$ spacer layer of the 3D sensor nanostructure, followed by $90 \mathrm{~nm}$ of PMMA, to have the top disk fully embedded at its center (see Figure $4 \mathrm{a}$ for the to-scale schematic). Combined analysis using atomic force microscopy (AFM) and ellipsometry confirms the flat and nonconformal profile of the obtained films, as well as their respective position along the height of the 3D sensor nanoarchitecture (Figure $4 \mathrm{~b}$ ). $T_{\mathrm{g}}$ determination is then carried out by heating the embedded metasurface under Ar flow (see Methods) and tracking individually the $\Delta \lambda_{\text {peak }}$ response of the two disks. Due to the thermal expansion of the polymers with increasing temperature, ${ }^{24}$ we observe that both $\Delta \lambda_{\text {peak }}$ signals initially blue shift linearly upon heating. The absolute magnitude of $\Delta \lambda_{\text {peak }}$ is slightly different due to the combination of slightly different BRIS of the top and bottom disks identified above and the thermal expansion coefficient of the polymers. Then, when the temperature reaches $\sim 115{ }^{\circ} \mathrm{C}$, the $\Delta \lambda_{\text {peak }}$ versus temperature trend of the top disk adopts a different slope, suggesting that the PMMA surrounding it has transformed from the "glassy" to a "rubbery" state ${ }^{24}$ and thus has undergone a glass transition characterized by $T_{\mathrm{g} \text { PMMA }}$. Interestingly, the $\Delta \lambda_{\text {peak }}$ evolution of the bottom disk is unaffected at this temperature as it proceeds to blue shift with a constant slope, until the temperature reaches $\sim 128{ }^{\circ} \mathrm{C}$. At this temperature, a distinct change in slope occurs (and vice versa the $\Delta \lambda_{\text {peak }}$ slope of top disk is constant), signifying the copolymer transition temperature, $T_{\mathrm{g}} \mathrm{P}$ (MMA-MAA). This finding has important implications as it clearly demonstrates the ability of the $3 \mathrm{D}$ sensor to measure individually and simultaneously the properties of the two stacked polymer thin films. In all of these cases, the derived $T_{\mathrm{g}}$ values are in excellent agreement with those in the literature for films of the same thicknesses, ${ }^{24,28,42,61,62}$ as well as with our own reference measurements using a conventional $2 \mathrm{D}$ indirect nanoplasmonic sensor $^{24}$ (Figure S15). To this end, the higher $T_{\mathrm{g}}$ in the copolymer is a manifestation of its stronger monomer bonds. ${ }^{60,61}$

To further validate the obtained $T_{\mathrm{g}}$ values and corroborate the independent sensing functionality of bottom and top disks in the $3 \mathrm{D}$ sensor metasurface, we carried out similar measurements for three other polymer layer configurations with identical total thickness: (i) PMMA-P(MMA-MAA) layered films, (ii) only P(MMA-MAA), and (iii) only PMMA (the last two cases have a single polymer layer covering both bottom and top disks; see Figure 4d). As summarized in Figure $4 \mathrm{~d}$, it is obvious that both disks in the sensor are able to accurately determine the $T_{\mathrm{g}}$ values of the polymers, irrespective of how they are arranged. Specifically, consistent $T_{\mathrm{g}}$ P(MMA-MAA) and $T_{\mathrm{g} \text { PMMA }}$ are found to be $\sim 128$ and $\sim 115^{\circ} \mathrm{C}$, respectively (raw data in Figure S16).
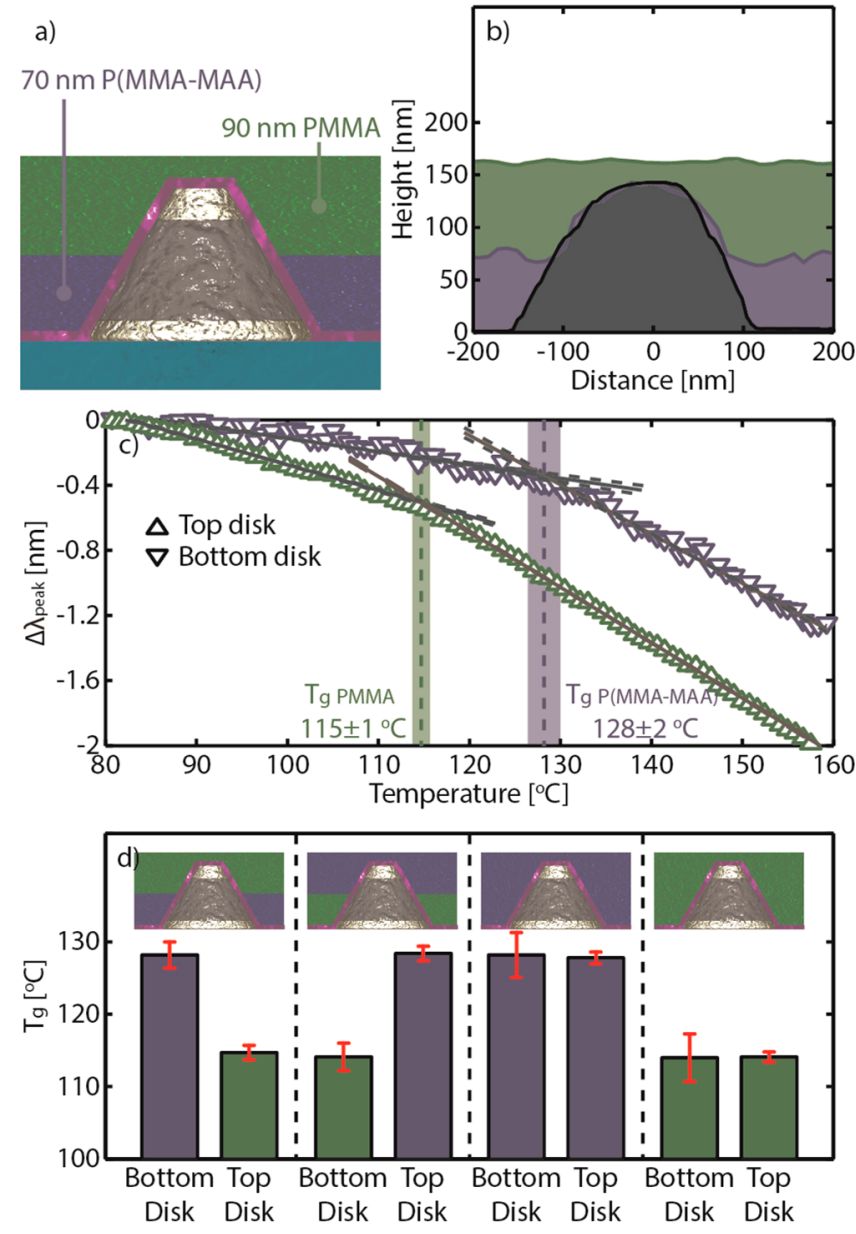

Figure 4. Glass transition temperature measurements of layered polymer thin films. (a) To-scale schematic of the studied system. The metasurface is coated with layered films of P(MMA-MAA) copolymer and PMMA, each encapsulating only one of the sensing disks. (b) AFM profiles of a single bare 3D sensor nanoarchitecture (black) and the same sensor covered with a layer of P(MMA-MAA) (blue) and PMMA (green). The profiles of the polymer layers are shifted vertically to their corresponding thicknesses measured using ellipsometry to mimic the actual configuration. Plotting the AFM data this way confirms that each disk is only encapsulated by one of the polymers in the layered structure. (c) Simultaneous $T_{\mathrm{g}}$ determination of the two polymer layers in the film by using the $\Delta \lambda_{\text {peak }}$ readouts obtained as a function of temperature from top and bottom disks. Solid and dashed gray lines represent linear fits to the data and corresponding $95 \%$ confidence intervals, respectively. A change in the slope indicates $T_{g}{ }^{24}$ as denoted by the colored dashed lines, with the error range related to the fitting marked by the colored areas. Distinct $T_{\mathrm{g}}$ is identified for both layers with that of copolymer being higher, in agreement with the literature. ${ }^{61}$ (d) $T_{\mathrm{g}}$ determined from the response of the top and bottom disks for various polymer layer configurations. Consistent $T_{\mathrm{g}}$ are found for all configurations. Error bars mark the $95 \%$ confidence interval of the fitting.

Finally, we highlight that our measurements above represent the state-of-the-art for successful simultaneous determination of the $T_{\mathrm{g}}$ of individual thin (i.e., a few tens to hundreds of nanometers) polymer films in a multilayer configuration. Previously, it was only possible to extract the $T_{\mathrm{g}}$ of only a single polymer layer in a stack. This was achieved by doping a fluorescent label into the polymer of interest, ${ }^{17,63,64}$ whose 
properties accordingly may have been altered by the presence of the label.

\section{CONCLUSIONS}

In conclusion, we have developed a plasmonic metasurface for nanoscale sensing in three dimensions, capable of detecting independently and simultaneously minute changes at two different locations in three-dimensional space. We achieved this function by vertically stacking in a 3D nanoarchitecture two plasmonic $\mathrm{Ag}$ nanodisks with dimensions sufficiently different to ensure spectrally well-separated LSPR modes in the visible-NIR range and by spatially putting them apart by a dielectric $\mathrm{SiO}_{2}$ spacer thick enough to decouple their nearfields. Integrating these nanostructures in a large-area quasirandom array on a flat surface generated a plasmonic metasurface for optical sensing in three dimensions. By further applying a thin conformal $\mathrm{Si}_{3} \mathrm{~N}_{4}$ coating to the metasurface, we were able to achieve both thermal and chemical stability in highly oxidizing conditions at up to $600{ }^{\circ} \mathrm{C}$ and uniform surface chemistry. Applying it to study the thermal properties of a thin polymer bilayer structure comprising stacked PMMA and P(MMA-MAA) films enabled the accurate and simultaneous determination of the glass transition temperatures of the individual thin polymer layers inside the stack.

Beyond the demonstrated application for characterization of thermal properties of multilayer thin polymer films, we predict numerous potential cases where our $3 \mathrm{D}$ sensing platform may be utilized, for example, characterization of nanomaterials that exhibit physical or chemical property gradients, ${ }^{17,65}$ diffusion/ intercalation processes in a (porous) matrix, ${ }^{66-68}$ and the discrimination of events occurring at different spatial locations (e.g., inside $v s$ outside) of a biological system. ${ }^{69-71}$ Furthermore, using an imaging technique capable of collecting optical spectra with in-plane spatial resolution (e.g., hyperspectral imaging), true spatially resolved 3D sensing can be realized. Finally, in a wider perspective, we hope that our work here will inspire further development of nanoplasmonic sensors that utilize multiple types of nanoparticles with spectrally distinguishable, and thus individually and simultaneously addressable, LSPR modes to facilitate applications where they are to function either individually ${ }^{18,19}$ or collectively. ${ }^{20}$

\section{METHODS}

Metasurface Nanofabrication. The details of the hole-mask colloidal lithography process undertaken to produce the sensors on both glass and silicon (e.g., materials, fabrication steps) is described elsewhere. ${ }^{72}$ Specific to the current work, the subsequent deposition of the $\mathrm{Ag}-\mathrm{SiO}_{2}-\mathrm{Ag}$ layers through the hole-mask was done in a Lesker PVD 225 evaporator with $1 \AA / \mathrm{s}$ deposition rate at a base pressure of $5 \times 10^{-7}$ Torr. The thin conformal $\mathrm{Si}_{3} \mathrm{~N}_{4}$ coating film was deposited in an STS PE-CVD system.

Metasurface Characterization. All SEM images were taken on surfaces fabricated on silicon and obtained in a Zeiss Supra $60 \mathrm{VP}$ with secondary electron detector, a working distance $<5 \mathrm{~mm}$, and an electron beam acceleration voltage of $5 \mathrm{kV}$. AFM profile measurements were done in tapping mode in air using an SPM Bruker Dimension 3100. Optical extinction spectra were collected using a Cary 5000 UV-vis-NIR spectrophotometer on samples fabricated on borofloat glass substrates.

Multilayer Thin Polymer Film Deposition. PMMA and $\mathrm{P}\left(\mathrm{MMA}(8.5)\right.$-MAA) (both from MicroChem, $\left.M_{\mathrm{w}}=950000\right)$ were utilized. We note that spin coating of the polymer with spinning parameters corresponding to the intended thickness resulted in the deposition of the polymer all over the sensor, including the 3D nanostructures in a conformal way. ${ }^{24,73}$ Thus, to obtain a flat polymer film with a protruding top part of the 3D nanostructure, a thick $(>500$ $\mathrm{nm}$ ) film was first spin coated on the sensor. The chosen thickness is deliberately much higher than the $3 \mathrm{D}$ sensor nanostructures to obtain a completely flat surface without protrusions due to the underlying nanostructures. After being baked at $170{ }^{\circ} \mathrm{C}$ for $5 \mathrm{~min}$, the film was then etched anisotropically using oxygen plasma $(50 \mathrm{~W}, 250 \mathrm{mTorr}$, Plasma-Therm Batchtop RIE 95m) down to the intended thickness, using an etch rate we previously determined $(\sim 2.45 \mathrm{~nm} / \mathrm{s}$ for both polymers). We note that the oxygen plasma transforms the $\mathrm{Si}_{3} \mathrm{~N}_{4}$ coating surface into $\mathrm{SiO}_{2}$ with thickness expected to depend on the etching details ${ }^{74,75}$ (e.g., $\mathrm{O}_{2}$ pressure, etching power, and duration). Subsequently, the second polymer was deposited in the same way. All of the described steps are illustrated in Figure S14.

Sensitivity Determination. Bulk refractive index sensitivity was determined by exposing the metasurface to mixtures of Milli- $\mathrm{Q}$ water (Millipore) and ethylene glycol (Sigma-Aldrich) at mixing ratios of 100:0, 80:20, 60:40, 40:60, and 20:80 wt \%, in a titanium flow cell (XNano, Insplorion $\mathrm{AB}$ ), under a constant flow of $100 \mu \mathrm{L} / \mathrm{min}$, as regulated by a peristaltic pump (Ismatec). The metasurface sensor was illuminated using a fiber-coupled halogen lamp (AvaLight-Hal, Avantes), whereas the extinction spectra were continuously recorded by a fiber-coupled fixed grating spectrometer (AvaSpec-HS-TEC, Avantes). To determine the decay lengths of the sensor, subsequent thin $\mathrm{Al}_{2} \mathrm{O}_{3}$ films were grown by atomic layer deposition (Oxford FlexAl). Intermittently, the film thickness (deposited on an analogue silicon chip simultaneously) was evaluated by ellipsometry (J.A. Woollam M2000), and the extinction spectra were recorded using a Cary 5000 spectrophotometer. Great care was taken to ensure that the spectra were always acquired from the same spot of the sample. The $\lambda_{\text {peak }}$ response was derived by fitting a 20th order polynomial to the spectra.

Oxygen Exposure and Elevated Temperature Experiments. The oxidation tests and $T_{\mathrm{g}}$ measurements were carried out in a quartz tube flow reactor with optical access (X1, Insplorion $A B)$. For the oxidation test, a total gas flow of $100 \mathrm{~mL} / \mathrm{min}$ was maintained using $\mathrm{Ar}$ as the carrier gas, with heating rate of $1{ }^{\circ} \mathrm{C} / \mathrm{min}$. For $T_{8}$ measurements, an Ar flow of $50 \mathrm{~mL} / \mathrm{min}$ with heating rate of 5 ${ }^{\circ} \mathrm{C} / \mathrm{min}$ was used. Prior to the $T_{\mathrm{g}}$ experiment, the sensors were annealed at $200{ }^{\circ} \mathrm{C}$ for $6 \mathrm{~h}$ to achieve a stable shape. This ensures no convolution of signal from the heat-induced reshaping of the sensors in the measurements (cf. Figure $2 b, c$ ). For both oxidation and $T_{g}$ experiments, the pressure in the reactor was atmospheric. The sample inside the flow reactor was illuminated by white light (AvaLight-Hal, Avantes) through an optical fiber coupled to a collimating lens. The transmitted light was then collected using a fiber-coupled fixed-grating spectrometer (AvaSpec-1024, Avantes). The $\lambda_{\text {peak }}$ response was derived by fitting a 20th order polynomial to the spectra.

FDTD Simulations. To simulate the optical response, FDTD simulations were performed using Lumerical FDTD Solutions. A single 3D sensor nanostructure was placed on a $\mathrm{SiO}_{2}$ substrate and consisted of four separate parts: a $\mathrm{Ag}$ bottom disk, a $\mathrm{SiO}_{2}$ spacer layer, a $\mathrm{Ag}$ top disk, and a $\mathrm{Si}_{3} \mathrm{~N}_{4}$ overlayer (see simulation geometry outlined in Figure 3a,b). To match the spectral position of the simulated plasmonic resonance to that of the measured one, we slightly varied the geometrical parameters. The bottom disk was simulated as a truncated cone with a taper angle of $26^{\circ}(200 \mathrm{~nm}$ bottom diameter and $185 \mathrm{~nm}$ top diameter and $20 \mathrm{~nm}$ thickness) with rounded corners ( $5 \mathrm{~nm}$ rounding). The spacer layer was simulated as a truncated cone extending $90 \mathrm{~nm}$ above the top of the bottom disk with a taper angle of $26^{\circ}$. The top disk was simulated as a truncated cone with a taper angle of $11^{\circ}(88 \mathrm{~nm}$ bottom diameter and 70 top diameter and $30 \mathrm{~nm}$ thickness) with rounded corners $(8 \mathrm{~nm}$ bottom and $20 \mathrm{~nm}$ top rounding). Finally, the $\mathrm{Si}_{3} \mathrm{~N}_{4}$ overlayer was simulated as a $10 \mathrm{~nm}$ thick layer following the underlying structure. To get correct field plots without artifacts, a mesh overlayer of $0.3 \mathrm{~nm}$ was used around the Ag disks and a rougher $1 \mathrm{~nm}$ mesh for the rest of the structure. Optical properties of the materials were taken from the literature with $\mathrm{SiO}_{2}$ set to a $\mathrm{RI}$ of $1.46, \mathrm{Si}_{3} \mathrm{~N}_{4}$ to a $\mathrm{RI}$ of 2.07 , the surrounding gas to a RI of 1 , and the dielectric function for Ag was 
taken from Hagemann et al. ${ }^{76}$ Light was introduced as a linearly polarized plane wave via a total-field/scattered-field source, and the scattering and absorption spectra were collected in all directions by integrating the Poynting vector of the field. The field intensity data were collected by power monitors at the resonance wavelengths of the two Ag disks.

\section{ASSOCIATED CONTENT}

\section{s. Supporting Information}

The Supporting Information is available free of charge at https://pubs.acs.org/doi/10.1021/acsnano.9b09508.

Experimental extinction spectra of 3D metasurfaces with various geometries, 3D metasurface array characteristics, SEM images of the 3D metasurfaces before and after oxidation/heating test, annealed 3D metasurface temperature response, combined electromagnetic field distribution at bottom and top disk resonant wavelengths, optical properties of $3 \mathrm{D}$ metasurfaces with reduced separation, raw data for BRIS and decay length measurements, sensitivity of metasurfaces with and without substrates, refractive index of the polymer thin films, deposition of flat multilayer thin films, control $T_{\mathrm{g}}$ measurements on conventional 2D plasmonic sensors, raw data for $T_{\mathrm{g}}$ measurements with metasurface 3D sensors (PDF)

\section{AUTHOR INFORMATION}

\section{Corresponding Authors}

Ferry Anggoro Ardy Nugroho - Department of Physics, Chalmers University of Technology, 41296 Göteborg, Sweden; (1) orcid.org/0000-0001-5571-0454;

Email: ferryanggoroardynugroho@yahoo.com

Christoph Langhammer - Department of Physics, Chalmers University of Technology, 41296 Göteborg, Sweden; (1) orcid.org/ 0000-0003-2180-1379; Email: clangham@chalmers.se

\section{Authors}

David Albinsson - Department of Physics, Chalmers University of Technology, 41296 Göteborg, Sweden; 이이.org/0000-00017275-6921

Tomasz J. Antosiewicz - Department of Physics, Chalmers University of Technology, 41296 Göteborg, Sweden; Faculty of Physics, University of Warsaw, 02-093 Warsaw, Poland; () orcid.org/0000-0003-2535-4174

Complete contact information is available at: https://pubs.acs.org/10.1021/acsnano.9b09508

\section{Notes}

The authors declare no competing financial interest.

\section{ACKNOWLEDGMENTS}

This research has received funding from the from the Knut and Alice Wallenberg Foundation Project 2016.0210, from the Swedish Foundation for Strategic Research Framework project RMA15-0052, from the European Research Council (ERC) under the European Union's Horizon 2020 research and innovation programme (678941/SINCAT), and the Polish National Science Center Project 2017/25/B/ST3/00744. Part of this work was carried out at the MC2 cleanroom facility and at the Chalmers Materials Analysis Laboratory. F.A.A.N. thanks Dr. Carl Wadell for assistance during the early phase of the project.

\section{REFERENCES}

(1) Bohren, C. F.; Huffman, D. R. Absorption and Scattering of Light by Small Particles; Wiley-VCH: New York, 2007.

(2) Linic, S.; Christopher, P.; Ingram, D. B. Plasmonic-Metal Nanostructures for Efficient Conversion of Solar to Chemical Energy. Nat. Mater. 2011, 10, 911-921.

(3) Linic, S.; Aslam, U.; Boerigter, C.; Morabito, M. Photochemical Transformations on Plasmonic Metal Nanoparticles. Nat. Mater. 2015, 14, 567-576.

(4) Stiles, P. L.; Dieringer, J. A.; Shah, N. C.; Van Duyne, R. P. Surface-Enhanced Raman Spectroscopy. Annu. Rev. Anal. Chem. 2008, $1,601-626$.

(5) Camden, J. P.; Dieringer, J. A.; Zhao, J.; Van Duyne, R. P. Controlled Plasmonic Nanostructures for Surface-Enhanced Spectroscopy and Sensing. Acc. Chem. Res. 2008, 41, 1653-1661.

(6) Mayer, K. M.; Hafner, J. H. Localized Surface Plasmon Resonance Sensors. Chem. Rev. 2011, 111, 3828-3857.

(7) Anker, J. N.; Hall, W. P.; Lyandres, O.; Shah, N. C.; Zhao, J.; Van Duyne, R. P. Biosensing with Plasmonic Nanosensors. Nat. Mater. 2008, 7, 442-453.

(8) Kedem, O.; Tesler, A. B.; Vaskevich, A.; Rubinstein, I. Sensitivity and Optimization of Localized Surface Plasmon Resonance Transducers. ACS Nano 2011, 5, 748-760.

(9) Englebienne, P. Use of Colloidal Gold Surface Plasmon Resonance Peak Shift to Infer Affinity Constants from the Interactions between Protein Antigens and Antibodies Specific for Single or Multiple Epitopes. Analyst 1998, 123, 1599-1603.

(10) Jackman, J. A.; Rahim Ferhan, A.; Cho, N.-J. Nanoplasmonic Sensors for Biointerfacial Science. Chem. Soc. Rev. 2017, 46, 36153660.

(11) Homola, J. Surface Plasmon Resonance Sensors for Detection of Chemical and Biological Species. Chem. Rev. 2008, 108, 462-493.

(12) Larsson, E. M.; Syrenova, S.; Langhammer, C. Nanoplasmonic Sensing for Nanomaterials Science. Nanophotonics 2012, 1, 249-266.

(13) Alekseeva, S.; Nedrygailov, I. I.; Langhammer, C. Single Particle Plasmonics for Materials Science and Single Particle Catalysis. ACS Photonics 2019, 6, 1319-1330.

(14) Langer, J.; Novikov, S. M.; Liz-Marzán, L. M. Sensing Using Plasmonic Nanostructures and Nanoparticles. Nanotechnology 2015, 26, 322001.

(15) Stewart, M. E.; Anderton, C. R.; Thompson, L. B.; Maria, J.; Gray, S. K.; Rogers, J. A.; Nuzzo, R. G. Nanostructured Plasmonic Sensors. Chem. Rev. 2008, 108, 494-521.

(16) Valsecchi, C.; Brolo, A. G. Periodic Metallic Nanostructures as Plasmonic Chemical Sensors. Langmuir 2013, 29, 5638-5649.

(17) Priestley, R. D.; Ellison, C. J.; Broadbelt, L. J.; Torkelson, J. M. Structural Relaxation of Polymer Glasses at Surfaces, Interfaces, and in Between. Science 2005, 309, 456-459.

(18) Sperling, J. R.; Macias, G.; Neale, S. L.; Clark, A. W. Multilayered Nanoplasmonic Arrays for Self-Referenced Biosensing. ACS Appl. Mater. Interfaces 2018, 10, 34774-34780.

(19) Rodrigo, D.; Tittl, A.; John-Herpin, A.; Limaj, O.; Altug, H. Self-Similar Multiresonant Nanoantenna Arrays for Sensing from Near- to Mid-Infrared. ACS Photonics 2018, 5, 4903-4911.

(20) Rodrigo, D.; Tittl, A.; Ait-Bouziad, N.; John-Herpin, A.; Limaj, O.; Kelly, C.; Yoo, D.; Wittenberg, N. J.; Oh, S. H.; Lashuel, H. A.; Altug, H. Resolving Molecule-Specific Information in Dynamic Lipid Membrane Processes with Multi-Resonant Infrared Metasurfaces. Nat. Commun. 2018, 9, 2160.

(21) Liu, N.; Hentschel, M.; Weiss, T.; Alivisatos, A. P.; Giessen, H. Three-Dimensional Plasmon Rulers. Science 2011, 332, 1407-1410.

(22) Dipalo, M.; Messina, G. C.; Amin, H.; La Rocca, R.; Shalabaeva, V.; Simi, A.; Maccione, A.; Zilio, P.; Berdondini, L.; De Angelis, F. 3D Plasmonic Nanoantennas Integrated with MEA Biosensors. Nanoscale 2015, 7, 3703-3711.

(23) De Angelis, F.; Malerba, M.; Patrini, M.; Miele, E.; Das, G.; Toma, A.; Zaccaria, R. P.; Di Fabrizio, E. 3D Hollow Nanostructures as Building Blocks for Multifunctional Plasmonics. Nano Lett. 2013, 13, 3553-3558. 
(24) Nugroho, F. A. A.; Diaz de Zerio Mendaza, A.; Lindqvist, C.; Antosiewicz, T. J.; Müller, C.; Langhammer, C. Plasmonic Nanospectroscopy for Thermal Analysis of Organic Semiconductor Thin Films. Anal. Chem. 2017, 89, 2575-2582.

(25) Diaz de Zerio Mendaza, A.; Melianas, A.; Nugroho, F. A. A.; Bäcke, O.; Olsson, E.; Langhammer, C.; Inganäs, O.; Müller, C. A Fullerene Alloy Based Photovoltaic Blend with a Glass Transition Temperature above $200{ }^{\circ} \mathrm{C}$. J. Mater. Chem. A 2017, 5, 4156-4162.

(26) Yu, L.; Qian, D.; Marina, S.; Nugroho, F. A. A.; Sharma, A.; Hultmark, S.; Hofmann, A. I.; Kroon, R.; Benduhn, J.; Smilgies, D. M.; Vandewal, K.; Andersson, M. R.; Langhammer, C.; Martín, J.; Gao, F.; Müller, C. Diffusion-Limited Crystallization: A Rationale for the Thermal Stability of Non-Fullerene Solar Cells. ACS Appl. Mater. Interfaces 2019, 11, 21766-21774.

(27) Jiang, N.; Sen, M.; Endoh, M. K.; Koga, T.; Langhammer, E.; Bjöörn, P.; Tsige, M. Thermal Properties and Segmental Dynamics of Polymer Melt Chains Adsorbed on Solid Surfaces. Langmuir 2018, 34, 4199-4209.

(28) Nugroho, F. A. A.; Frost, R.; Antosiewicz, T. J.; Fritzsche, J.; Larsson Langhammer, E. M.; Langhammer, C. Topographically Flat Nanoplasmonic Sensor Chips for Biosensing and Materials Science. ACS Sensors 2017, 2, 119-127.

(29) Firdaus, Y.; He, Q.; Lin, Y.; Nugroho, F. A. A.; Le Corre, V. M.; Yengel, E.; Balawi, A. H.; Seitkhan, A.; Laquai, F.; Langhammer, C.; Liu, F.; Heeney, M.; Anthopoulos, T. D. Novel Wide-Bandgap NonFullerene Acceptors for Efficient Tandem Organic Solar Cells. J. Mater. Chem. A 2020, 8, 1164-1175.

(30) Johnson, P. B.; Christy, R. W. Optical Constants of the Noble Metals. Phys. Rev. B 1972, 6, 4370-4379.

(31) Yang, H. U.; D’Archangel, J.; Sundheimer, M. L.; Tucker, E.; Boreman, G. D.; Raschke, M. B. Optical Dielectric Function of Silver. Phys. Rev. B: Condens. Matter Mater. Phys. 2015, 91, 235137.

(32) Lee, K.-S.; El-Sayed, M. A. Gold and Silver Nanoparticles in Sensing and Imaging: Sensitivity of Plasmon Response to Size, Shape, and Metal Composition. J. Phys. Chem. B 2006, 110, 19220-19225.

(33) Zorić, I.; Zäch, M.; Kasemo, B.; Langhammer, C. Gold, Platinum, and Aluminum Nanodisk Plasmons: Material Independence, Subradiance, and Damping Mechanisms. ACS Nano 2011, 5, $2535-2546$

(34) Fredriksson, H.; Alaverdyan, Y.; Dmitriev, A.; Langhammer, C.; Sutherland, D. S.; Zäch, M.; Kasemo, B. Hole-Mask Colloidal Lithography. Adv. Mater. 2007, 19, 4297-4302.

(35) Syrenova, S.; Wadell, C.; Langhammer, C. Shrinking-Hole Colloidal Lithography: Self-Aligned Nanofabrication of Complex Plasmonic Nanoantennas. Nano Lett. 2014, 14, 2655-2663.

(36) Dmitriev, A.; Pakizeh, T.; Käll, M.; Sutherland, D. S. GoldSilica-Gold Nanosandwiches: Tunable Bimodal Plasmonic Resonators. Small 2007, 3, 294-299.

(37) Jain, P. K.; El-Sayed, M. A. Plasmonic Coupling in Noble Metal Nanostructures. Chem. Phys. Lett. 2010, 487, 153-164.

(38) Antosiewicz, T. J.; Apell, S. P.; Zäch, M.; Zorić, I.; Langhammer, C. Oscillatory Optical Response of an Amorphous Two-Dimensional Array of Gold Nanoparticles. Phys. Rev. Lett. 2012, 109, 247401.

(39) Antosiewicz, T. J.; Apell, S. P. Plasmonic Glasses: Optical Properties of Amorphous Metal-Dielectric Composites. Opt. Express 2014, 22, 2031-2042.

(40) Antosiewicz, T. J.; Tarkowski, T. Localized Surface Plasmon Decay Pathways in Disordered Two-Dimensional Nanoparticle Arrays. ACS Photonics 2015, 2, 1732-1738.

(41) Czajkowski, K. M.; Antosiewicz, T. J. Electromagnetic Coupling in Optical Devices Based on Random Arrays of Dielectric Nanoresonators. J. Phys. Chem. C 2020, 124, 896-905.

(42) Langhammer, C.; Larsson, E. M.; Kasemo, B.; Zorić, I. Indirect Nanoplasmonic Sensing: Ultrasensitive Experimental Platform for Nanomaterials Science and Optical Nanocalorimetry. Nano Lett. 2010, 10, 3529-3538.

(43) Riley, F. L. Silicon Nitride and Related Materials. J. Am. Ceram. Soc. 2000, 83, 245-265.
(44) Kuzma, A.; Weis, M.; Flickyngerova, S.; Jakabovic, J.; Satka, A.; Dobrocka, E.; Chlpik, J.; Cirak, J.; Donoval, M.; Telek, P.; Uherek, F.; Donoval, D. Influence of Surface Oxidation on Plasmon Resonance in Monolayer of Gold and Silver Nanoparticles. J. Appl. Phys. 2012, 112, 103531.

(45) Orendorff, C. J.; Sau, T. K.; Murphy, C. J. Shape-Dependent Plasmon-Resonant Gold Nanoparticles. Small 2006, 2, 636-639.

(46) Liz-Marzán, L. M.; Mulvaney, P. Au@SiO2 Colloids: Effect of Temperature on the Surface Plasmon Absorption. New J. Chem. 1998, $22,1285-1288$.

(47) Link, S.; El-Sayed, M. A. Size and Temperature Dependence of the Plasmon Absorption of Colloidal Gold Nanoparticles. J. Phys. Chem. B 1999, 103, 4212-4217.

(48) Dmitriev, A.; Hägglund, C.; Chen, S.; Fredriksson, H.; Pakizeh, T.; Käll, M.; Sutherland, D. S. Enhanced Nanoplasmonic Optical Sensors with Reduced Substrate Effect. Nano Lett. 2008, 8, 38933898.

(49) Aćimović, S. S.; Ś́pová, H.; Emilsson, G.; Dahlin, A. B.; Antosiewicz, T. J.; Käll, M. Superior LSPR Substrates Based on Electromagnetic Decoupling for On-a-Chip High-Throughput LabelFree Biosensing. Light: Sci. Appl. 2017, 6, e17042-e17042.

(50) Kvasnička, P.; Homola, J. Optical Sensors Based on Spectroscopy of Localized Surface Plasmons on Metallic Nanoparticles: Sensitivity Considerations. Biointerphases 2008, 3, FD4FD11.

(51) Miller, M. M.; Lazarides, A. A. Sensitivity of Metal Nanoparticle Surface Plasmon Resonance to the Dielectric Environment. J. Phys. Chem. B 2005, 109, 21556-21565.

(52) Mazzotta, F.; Johnson, T. W.; Dahlin, A. B.; Shaver, J.; Oh, S. H.; Höok, F. Influence of the Evanescent Field Decay Length on the Sensitivity of Plasmonic Nanodisks and Nanoholes. ACS Photonics 2015, 2, 256-262.

(53) Kroschwitz, J. I. Encyclopedia of Polymer Science and Engineering; John Wiley \& Sons, Ltd.: New York, 1990.

(54) Dalal, S. S.; Walters, D. M.; Lyubimov, I.; De Pablo, J. J.; Ediger, M. D. Tunable Molecular Orientation and Elevated Thermal Stability of Vapor-Deposited Organic Semiconductors. Proc. Natl. Acad. Sci. U. S. A. 2015, 112, 4227-4232.

(55) Ràfols-Ribé, J.; Will, P. A.; Hänisch, C.; Gonzalez-Silveira, M.; Lenk, S.; Rodríguez-Viejo, J.; Reineke, S. High-Performance Organic Light-Emitting Diodes Comprising Ultrastable Glass Layers. Sci. Adv. 2018, 4, No. eaar8332.

(56) Kim, J. Y.; Lee, K.; Coates, N. E.; Moses, D.; Nguyen, T. Q.; Dante, M.; Heeger, A. J. Efficient Tandem Polymer Solar Cells Fabricated by All-Solution Processing. Science 2007, 317, 222-225.

(57) You, J.; Dou, L.; Yoshimura, K.; Kato, T.; Ohya, K.; Moriarty, T.; Emery, K.; Chen, C.-C.; Gao, J.; Li, G.; Yang, Y. A Polymer Tandem Solar Cell with $10.6 \%$ Power Conversion Efficiency. Nat. Commun. 2013, 4, 1446.

(58) Müller, C. On the Glass Transition of Polymer Semiconductors and Its Impact on Polymer Solar Cell Stability. Chem. Mater. 2015, 27, $2740-2754$.

(59) Bertho, S.; Haeldermans, I.; Swinnen, A.; Moons, W.; Martens, T.; Lutsen, L.; Vanderzande, D.; Manca, J.; Senes, A.; Bonfiglio, A. Influence of Thermal Ageing on the Stability of Polymer Bulk Heterojunction Solar Cells. Sol. Energy Mater. Sol. Cells 2007, 91, 385-389.

(60) Huang, C.-F.; Chang, F.-C. Comparison of Hydrogen Bonding Interaction between PMMA/PMAA Blends and PMMA-Co-PMAA Copolymers. Polymer 2003, 44, 2965-2974.

(61) Kuo, S.-W.; Kao, H.-C.; Chang, F.-C. Thermal Behavior and Specific Interaction in High Glass Transition Temperature PMMA Copolymer. Polymer 2003, 44, 6873-6882.

(62) Porter, C. E.; Blum, F. D. Thermal Characterization of PMMA Thin Films Using Modulated Differential Scanning Calorimetry. Macromolecules 2000, 33, 7016-7020.

(63) Ellison, C. J.; Torkelson, J. M. The Distribution of GlassTransition Temperatures in Nanoscopically Confined Glass Formers. Nat. Mater. 2003, 2, 695-700. 
(64) Roth, C. B.; Torkelson, J. M. Selectively Probing the Glass Transition Temperature in Multilayer Polymer Films: Equivalence of Block Copolymers and Multilayer Films of Different Homopolymers. Macromolecules 2007, 40, 3328-3336.

(65) Kim, M. S.; Khang, G.; Lee, H. B. Gradient Polymer Surfaces for Biomedical Applications. Prog. Polym. Sci. 2008, 33, 138-164.

(66) Gusak, V.; Heiniger, L.-P.; Graetzel, M.; Langhammer, C.; Kasemo, B. Time-Resolved Indirect Nanoplasmonic Sensing Spectroscopy of Dye Molecule Interactions with Dense and Mesoporous TiO2 Films. Nano Lett. 2012, 12, 2397-2403.

(67) Kreno, L. E.; Hupp, J. T.; Van Duyne, R. P. Metal-Organic Framework Thin Film for Enhanced Localized Surface Plasmon Resonance Gas Sensing. Anal. Chem. 2010, 82, 8042-8046.

(68) Nugroho, F. A. A.; Xu, C.; Hedin, N.; Langhammer, C. UVVisible and Plasmonic Nanospectroscopy of the CO2 Adsorption Energetics in a Microporous Polymer. Anal. Chem. 2015, 87, 1016110165.

(69) Caprettini, V.; Huang, J. A.; Moia, F.; Jacassi, A.; Gonano, C. A.; Maccaferri, N.; Capozza, R.; Dipalo, M.; De Angelis, F. Enhanced Raman Investigation of Cell Membrane and Intracellular Compounds by 3D Plasmonic Nanoelectrode Arrays. Adv. Sci. 2018, 5, 1800560.

(70) Tian, B.; Cohen-Karni, T.; Qing, Q.; Duan, X.; Xie, P.; Lieber, C. M. Three-Dimensional, Flexible Nanoscale Field-Effect Transistors as Localized Bioprobes. Science 2010, 329, 830-834.

(71) Almquist, B. D.; Melosh, N. A. Fusion of Biomimetic Stealth Probes into Lipid Bilayer Cores. Proc. Natl. Acad. Sci. U. S. A. 2010, 107, 5815-5820.

(72) Nugroho, F. A. A.; Darmadi, I.; Cusinato, L.; Susarrey-Arce, A.; Schreuders, H.; Bannenberg, L. J.; da Silva Fanta, A. B.; Kadkhodazadeh, S.; Wagner, J. B.; Antosiewicz, T. J.; Hellman, A.; Zhdanov, V. P.; Dam, B.; Langhammer, C. Metal-Polymer Hybrid Nanomaterials for Plasmonic Ultrafast Hydrogen Detection. Nat. Mater. 2019, 18, 489-495.

(73) Hamans, R. F.; Parente, M.; Castellanos, G. W.; Ramezani, M.; Gómez Rivas, J.; Baldi, A. Super-Resolution Mapping of Enhanced Emission by Collective Plasmonic Resonances. ACS Nano 2019, 13, $4514-4521$.

(74) Kennedy, G. P.; Buiu, O.; Taylor, S. Oxidation of Silicon Nitride Films in an Oxygen Plasma. J. Appl. Phys. 1999, 85, 33193326.

(75) Jiménez, C.; Perrière, J.; Vickridge, I.; Enard, J. P.; Albella, J. M. Transformation of Silicon Nitride in Oxygen Plasma. Surf. Coat. Technol. 1991, 45, 147-154.

(76) Hagemann, H. J.; Gudat, W.; Kunz, C. Optical Constants from the Far Infrared to the X-Ray Region: $\mathrm{Mg}, \mathrm{Al}, \mathrm{Cu}, \mathrm{Ag}, \mathrm{Au}, \mathrm{Bi}, \mathrm{C}$, and Al2O3. J. Opt. Soc. Am. 1975, 65, 742-744. 